\title{
Modelling the mock-up: towards the automatic specification of the behaviour of early prototypes
}

\author{
Chris Phillips and Chris Scogings
}

\author{
Department of Computer Science \\ Massey University \\ New Zealand \\ \{C.Phillips, C.Scogings\}@massey.ac.nz
}

\begin{abstract}
A shortcoming of current early prototyping tools is that although they permit the designer to construct a mock-up of the look and feel of the interface, they provide no model of the interaction. Such tools could be extended to produce such a model as a byproduct of the construction of the prototype. This research explores such an extension to HyperCard, and reviews its utility. The interaction model is specified in Lean Cuisine+, a semi-formal graphical notation for describing the behaviour of direct manipulation graphical user interfaces.
\end{abstract}

KEY WORDS early prototyping, interaction model, HyperCard, Lean Cuisine+

\section{INTRODUCTION}

Prototyping supports the view that good interfaces can only be constructed through iterative refinement. It involves constructing a smaller scale version or mockup of a proposed system. A variety of prototyping tools and techniques are available to support iterative interface development, from pencil and paper mockups to full scale interface development environments (Hix \& Schulman, 1991; Myers, 1993; Szekely, 1994).

The focus of this research is on support for early prototyping. A shortcoming of current early prototyping tools is that although they permit the designer to construct a mock-up of the look and feel of the interface, they provide no model of the interaction. The mock-up exists and can be exercised, but no specification or description of the interaction exists outside the code produced. The focus is rather on 'screens', i.e. on the interface rather than the interaction.

Early prototyping tools could be extended to produce a model of the interaction as a byproduct of the construction of the prototype. This research explores such an extension to HyperCard, and briefly reviews its utility. The interaction model is specified in Lean Cuisine+ (Phillips, 1995), a semi-formal graphical notation for describing the behaviour of direct manipulation graphical user interfaces.

\section{EXTENDING HYPERCARD}

HyperCard (Claris, 1992) is based on two primary abstractions: cards and stacks (of cards). Each card corresponds to one screen, and may contain buttons and text fields in addition to artwork. Backgrounds may be defined to contain features common to several cards. Each HyperCard object (button, field, stack etc) has an associated HyperTalk script which describes what it must do in response to messages (e.g. mouse clicks). Using HyperCard, the designer may define the appearance of screens, and also simulate the behaviour of menus, buttons etc in linking screens. Thus both the look and feel of the interface can be created.

A HyperCard stack has been developed to prototype an interface for a real estate application in which potential house buyers can select likely properties from 
a data base of the properties currently for sale in a given town. The 'Property' Stack consists of the following cards, each of which represents one screen in the prototype:

- A Town Map Card: Which shows a map of the town with the location of properties marked. Properties can be selected by direct manipulation.

- A Search Card: Which provides fields for the entering of selection criteria, and buttons for initiating the selection of properties.

- Property Cards: Each of which displays details of one property, and provides buttons for continuing the search.

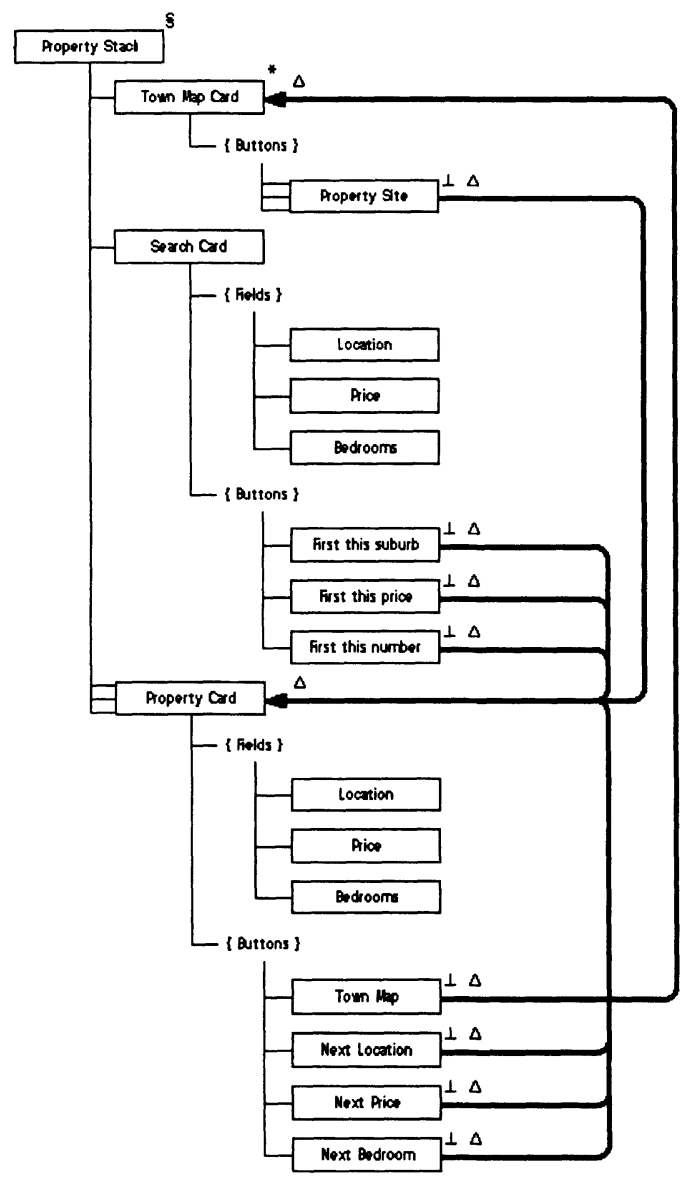

Figure 1: Property Stack: Lean Cuisine + diagram
The Lean Cuisine+ specification of this stack is shown in Figure 1. It has been produced semiautomatically from the associated HyperTalk scripts. The stack is represented by a dialogue tree, which shows inter-relationships and some of the constraints which impact the behaviour of the selectable primitives. Further constraints are captured in the form of selection triggers, represented by directed arcs.

\section{REVIEW}

A semi-formal model of the interaction associated with a HyperCard stack has been produced in the form of a graphical specification using Lean Cuisinet. The model provides information on the interaction which could otherwise only be uncovered by exercising the stack and committing the behaviour to memory. This information provides useful documentation, and can be analysed for structural shortcomings and inconsistency within the dialogue. For example, excessive navigation between screens in performing tasks can be uncovered.

HyperCard exhibits generic features of prototyping tools for GUIs - the representation of objects (including screens) and the links between them. The graphical modelling being explored in this research should thus be applicable to a wider range of interface development tools.

\section{REFERENCES}

Hix, D. \& Schulman, R.S. (1991): Human-Computer Interface Development Tools: A Methodology for their Evaluation, Comms. $A C M, 34,3,74-87$.

HyperCard (1992): Claris Corporation.

Myers, B.A. (1993): State of the Art in User Interface Tools, In Hartson, H.R. and Hix, D. (Eds), Advances in Human-Computer Interaction, Ablex, Norwood, NJ, 11-28.

Phillips, C.H.E. (1995): Lean Cuisinet: An executable graphical notation for describing direct manipulation interfaces, Interacting with Computers, 7, 1, 1995, 49-71.

Szekely, P. (1994): User Interface Prototyping: Tools and Techniques, Proc. ICSE'94 Workshop on SEHCI, Sorrento, Italy, Springer, 76-92. 